\title{
Lipidomics Reveals Glycerophospholipid \\ Metabolism Dysregulation in the Corpus Striatum of Mice Treated with Cefepime
}

Xiaocong Liu ( $\sim$ xiaocongliu2021@163.com )

Sichuan University https://orcid.org/0000-0002-5837-1985

Xiaojie Wang

Sichuan University

Qingfan Wei

Sichuan University

Xiaowei Yang

Sichuan University

Jiamei Zhang

Sichuan University

Rui Xu

Sichuan University

Haoluo Zhang

Sichuan University

Shaomin Wang

Sichuan University

Xuemei Wan

Sichuan University

Linhong Jiang

Sichuan University

Yuman He

Sichuan University

Shu Li

Sichuan University

Rong Chen

Sichuan University

Yonghai Wang

Sichuan University

Yaxing Chen

Sichuan University

Feng Qin 
Sichuan University

\section{Yuanyuan Chen}

Sichuan University West China Hospital

\section{Yanping Dai}

Sichuan University

\section{Hongchun Li}

Sichuan University

\section{Ying Zhao}

Sichuan University

Huaqin Zhang

Sichuan University

\section{Qian Bu}

Sichuan University

\section{Hongbo Wang}

Sichuan University

Jingwei Tian

Sichuan University

\section{Yinglan Zhao}

Sichuan University

\section{Xiaobo Cen}

Sichuan University

\section{Research Article}

Keywords: Cefepime, Lipidomics, Corpus Striatum, Glycerophospholipids

Posted Date: April 8th, 2021

DOl: https://doi.org/10.21203/rs.3.rs-350165/v1

License: (c) (1) This work is licensed under a Creative Commons Attribution 4.0 International License. Read Full License 


\section{Abstract}

Cefepime exhibits a broad spectrum of antimicrobial activity and thus is widely used for severe bacterial infection. Adverse effects on the central nervous system (CNS) have been reported in the patients treated with cefepime. Current explanation for the neurobehavioral effect of cefepime mainly attributes to its ability to across blood-brain barrier and to competitively bind to GABAergic receptor; however, the underlying mechanism is largely unknown. In this study, mice were intraperitoneally administered 80 $\mathrm{mg} / \mathrm{kg}$ cefepime for different time period, including 1 day, 3 days, 5 days, 7 days and 10 days, and then LC/MS-MS-based metabolomics was used to investigate the effect of cefepime on the brain lipidomic profiling and metabolic pathway. Repeated cefepime treatment time-dependently caused anxiety-like behavior accompanied with the reduced locomotor activity in the open field test. Cefepime profoundly altered the lipid profile in the corpus striatum, and glycerophospholipid contributed to a large proportion among those significantly modified lipids. Cefepime also significantly modified acyl chain length and unsaturation of fatty acids. In addition, cefepime obviously altered the morphology of neurite, mitochondria and synaptic vesicles of striatal neuron in vitro. Collectively, our results show that cefepime reprograms glycerophospholipid metabolism of corpus striatum, which may underly its neurobehavioral effect.

\section{Introduction}

Cefepime is the fourth-generation cephalosporin antibiotic and a broad-spectrum antibiotic applying to hospitalized patients with a range of infections. However, about $10 \sim 23 \%$ of patients have been discovered aberrant nervous system-related behavior on average four to five days after starting cefepime therapy. The most frequently encountered symptoms are the reduced consciousness, myoclonus, confusion, aphasia, seizures, agitation (Honore and Spapen. 2015; Kamboj et al. 2020; Khasani, 2015; Payne et al. 2017). The proposed pathophysiology for these adverse effects of cefepime has been attributed to its ability to across the blood-brain barrier (BBB) and competitively bind to the $y$-amino butyric acid (GABA) receptors, eventually leading to the suppression of inhibitory neurotransmission (Amakhin et al. 2018; Bhattacharyya et al. 2016; Payne et al. 2017). Risks factors of cefepime include excessive dosing, enhanced serum level, renal insufficiency, preexisting brain injury, patients with hematological malignancy or underwent intensive care during hospitalization. Occasionally, the adverse side effects still occur despite appropriate cefepime application in clinic. The mechanism underlying the adverse event of cefepime is still largely unknown.

Brain is the second highest lipid-enriched organ behind adipose tissue, and total lipids mass contribute to the half of brain dry weight. Diverse lipids play pleiotropic crucial roles in the brain, ranging from the backbone for biological membrane, to cell signaling molecules, and regulation of immune responses (Beaulieu et al. 2019; Gantz and Bean, 2017; Manni et al. 2018; Mesa-Herrera et al. 2019). Impairment of lipid homeostasis is involved in the pathology of central nervous system (CNS) disorders and neurodegenerative diseases, such as Alzheimer's disease (AD), Parkinson's disease (PD) (Fang et al. 2019; Kunkle et al. 2019; Valadas et al. 2018). For instance, in AD brain, 4-hydroxynonenal (4-HNE) and 4- 
hydroxy hexenal (4-HHE), the aldehydic products of arachidonic acid and docosahexaenoic acid (DHA) metabolism, are accumulated into intraneuronal neurofibrillary tangles, contributing to the derangement of cytoskeleton structure in neurons (Castro et al. 2017; Rao et al. 2011; Yassine et al. 2017). The interaction of a-synuclein with free forms of polyunsaturated fatty acids (PUFA) elevates the propensity to the aggregation of a-synuclein, contributing to the progress of PD (Fanning et al. 2019; Fecchio et al. 2018; Imberdis et al. 2019).

Phospholipids are amphipathic molecules with so-called polar head group and nonpolar tail ends. The non-polar tails contain hydrocarbon chains that vary in the chain length of fatty acid, double bond number and position. These characters determine the structure and geometry of fatty acyl chains, eventually governing the shape of lipids, the degree of lipid packing within bilayer, and membrane fluidity. Membrane fluidity is a key parameter for membrane fusion, influencing lipid mobility and protein function. Disturbance of glycerophospholipids metabolism in the neural membrane remarkably alters the activities of membrane-bound proteins, such as enzymes, receptors and ion channels (Hanada, 2018; Serricchio et al. 2018; Wang and Tontonoz, 2019). Disorder of lipids composition, geometry, saturation or distribution drives the impairment the neuronal function, neurotransmitter signals as well as synaptic transmission (loannou et al. 2019; Shirane et al. 2020; Yang et al. 2020). Increasing studies have found a link between membrane physicochemical state and pathologies (Diaz and Retamal, 2018; Sweeney et al. 2018). For instance, the decreases of monounsaturated oleic acid (18:1), DHA and PUFA at early stages of $A D$ cause higher molecular order and microviscosity of lipid raft. Pathophysiological consequences of these alterations are the accumulation of $\beta$-secretase within lipid rafts, thereby favoring the amyloidogenic process of amyloid precursor protein (APP) (Díaz et al. 2015; Mesa-Herrera et al. 2019).

In the present study, we performed unbiased lipidomic analysis to explore the effect of cefepime on neurobehaviors in mice. We found that several classes of lipids in the corpus striatum was profoundly altered after cefepime exposure for 5 continuous days, and glycerophospholipids took a large proportion among those modified lipids. In combination with the findings from the cultured striatal neurons exposed to cefepime, we propose that dysregulated glycerophospholipid metabolism may contribute to CNS adverse effect of cefepime in mice.

\section{Materials And Methods}

\subsection{Drugs}

Cefepime was purchased from Selleck Chemicals (Houston, TX) and dissolved in 5\% DMSO, 30\% PEG300 and $65 \%$ pure water.

\subsection{Animals}

Adult male C57BL/6 mice (6-8 weeks, body weight 18-22 g) were purchased from Vital River Laboratory Animal Technology Co. Ltd (Beijing, China). All mice were housed under standard condition (a 12-h light/12-h dark cycle with light on from 07:00-19:00) with a constant room temperature. All experimental 
procedures and use of the animals were conducted in accordance with the guidelines established by the Association for Assessment and Accreditation of Laboratory Animal Care and the Institutional Animal Care and Use Committee of Sichuan University.

\subsection{Drug Injection}

Mice were intraperitoneally administered $80 \mathrm{mg} / \mathrm{kg}$ cefepime for different time period. This dosage was based on the pharmacokinetic study of cefepime single regimen and mono-therapeutic dose in murine model (Bhagwat et al. 2019; Pechère and Vladoianu, 1992).

\subsection{Open field test (OFT)}

All experiments were carried out in a dark cycle, started from 90 minutes turned off the lights, and arrived at the test room after at least 2 hours of adaptation time. This test schedule and method followed previously published research (Tomihara et al. 2009). Open filed test was applied to check the movement activity and anxiety-like behavior. A 16-beam animal activity monitor was used to divide the black acrylic box $(48 \times 48 \times 30 \mathrm{~cm}$ ) into center and periphery. EthoVision version 7.0 software (Noldus Information Technology) was applied to analyze a series of parameters basing on the recorded activity, including total movement distance, time spent in the inner zone $(24 \times 24 \times 30 \mathrm{~cm})$ and the number of entries into the inner zone. The movement time only refers to the time consumed by the animal moving but exclude the time without moving. At the beginning of first adaption, mouse was placed in the same position (rear left corner) of the arena and allowed to spontaneously move in the open field arena for 5 minutes.

\subsection{Blood collection and tissue isolation}

Mice were immediately killed by rapid decapitation after the end point of open field test. Blood was collected for the measurement of serum biochemistry. Brain tissue and other major organs were subsequently dissociated out for lipidomic analysis and histopathological evaluation. For lipidomic profiling analysis, the isolated cerebral cortex, hippocampus and corpus striatum were quickly frozen via dipping into liquid nitrogen. The frozen tissues were stored at $-80^{\circ} \mathrm{C}$ until lipid extraction.

Histopathological assessment was performed through HE staining. Heart, liver, spleen, lung, kidney and whole brain of the mouse were dissociated and fixed in a $4 \%$ paraformaldehyde solution, followed by tissue sectioning and HE staining.

\subsection{Blood biochemical test}

A series of blood chemical parameters were detected using the purified serum without any contamination of blood cells. Briefly, the collected fresh blood samples were purified through centrifuging at $1500 \mathrm{rpm}$ for $10 \mathrm{~min}$ and the serum in the upper layer was aspirated for further test. The biochemical parameters were assessed using an automatic biochemical analyzer (Cobas c311, Switzerland). The following parameters, the serum alanine aminotransferase (ALT), serum albumin (ALB), creatine kinase isoenzyme (CK-MB), alkaline phosphatase (ALP), aspartate aminotransferase (AST), serum total protein (TP), cholesterol (CHOL), uric acid (UA), urea, lactate dehydrogenase (LDH), triglyceride (TG), direct bilirubin (DBiL) and serum creatinine (Cre) were measured. 


\subsection{HE staining}

The major organs, including heart, liver, spleen, lung, kidney and brain, were exteriorized and fixed in $4 \%$ paraformaldehyde solution. The fixed tissue was embedded with paraffin, and was further sectioned with a thickness of $5 \mu \mathrm{m}$ after dewaxing and rehydration. The sections were stained with hematoxylin solution for 5 minutes, and then immersed in $1 \%$ acidic ethanol for another 5 times. The sections were then counterstained with eosin-phloxine solution for 1 minutes, and dehydrated with a gradient alcohol and cleared with in xylene.

\subsection{Lipid extraction}

Lipids extracts from mouse brain were prepared basing on methyl tert-butyl ether (MTBE) lipid extraction methods. Briefly, pre-chilled methanol $(150 \mu \mathrm{l})$ and MTBE $(450 \mu \mathrm{l})$ were sequentially added into the frozen tissue (25-30 mg). The mixture was incubated at room temperature for 10 minutes and subsequently homogenized at 6,500 rpm in three cycles for $15 \mathrm{~s}$ with 20s intervals using a bead-based homogenizer (Precellys Evolution, Bertin Technologies, Montigny le Bretonneux, France). Then, aspirate $300 \mu \mathrm{l}$ of $25 \%$ methanol diluted in sterile MiliQ water and add it into the homogeneous mixture in order to form a phase separation. Centrifuge at $14,000 \mathrm{~g}$ for 10 minutes after vigorous mixing. Aspirate the upper organic phase carefully without any disturbing the middle layer and transfer it into another tube. The extracted lipids were evaporated at room temperature under a gentle stream of nitrogen and stored at $-80^{\circ} \mathrm{C}$ until use.

\subsection{Mass spectrometry analysis of lipid metabolites}

Lipid analysis was performed on an Acquity Ultra Performance Liquid Chromatography (UPLC system) coupled with hybrid quadrupole orthogonal time-of-flight lass Spectrometer (Waters, Milford, MA, USA). Electrospray positive and negative ionization modes were used. In detail, the extracted lipids powder was firstly re-dissolved in acetonitrile/isopropanol $(v / v, 7: 3)$. Then, the injected lipids were separated through Waters Acquity HSS T3 Column (Waters, Milford, MA, USA) with a constant temperature at $55^{\circ} \mathrm{C}$ in column oven. The average column pressures were maintained around ca. 10,000 psi. Elution buffer $A$ consists of acetonitrile and water with $10 \mathrm{mM}$ ammonium acetate $(40: 60, \mathrm{v} / \mathrm{v})$, and elution buffer $B$ consists of acetonitrile and isopropanol $(10: 90, \mathrm{v} / \mathrm{v})$ with $10 \mathrm{mM}$ ammonium acetate. The sample analysis was performed by a binary gradient system over $15 \mathrm{~min}$ total run time. At the start point of the gradient, it was held at $60 \%$ elution buffer $A$ and $40 \%$ elution buffer $B$. In the following 10 min, the gradient was ramped in a linear fashion to $100 \%$ elution buffer $B$ and held at this composition for another 2 min. The system was switched back to $60 \%$ elution buffer B and $40 \%$ elution buffer $A$, and equilibrated for an additional $3 \mathrm{~min}$. The flow rate was set at $0.4 \mathrm{~mL} / \mathrm{min}$ and the injection volume was $10 \mu \mathrm{l}$.

The capillary voltage and cone voltage were separately set at $3.0 \mathrm{kV}$ and $40 \mathrm{~V}$ in positive mode. On contrary to positive mode, change the cone voltage to $-40 \mathrm{~V}$ while maintain the same capillary voltage under the negative ion detection mode. The temperature of resolvent gas was kept at $300^{\circ} \mathrm{C}$, and the speed of resolvent gas and column gas was set at $600 \mathrm{~L} / \mathrm{h}$ and $50 \mathrm{~L} / \mathrm{h}$, respectively. The spray was used to ensure the accuracy of the results. Leucine $(4 \mathrm{ng} / \mathrm{ml})$ was used in positive ion mode $\left([\mathrm{M}+\mathrm{H}]^{+}\right.$ 
$=556.2771)$ and negative ion mode $\left.(\mathrm{M}+\mathrm{H}]^{-}=554.2615\right)$. MassLynx (Milford, Massachusetts, USA) was used to collect data in continuous mode.

\subsection{Data processing and analysis}

Progenesis QI software (Newcastle, UK) was used to process UPLC-ESI-TOFMS data. Data were sequentially processed through comparison, peak selection and lipid identification. We identified the lipid metabolites by referring primarily to the lipid map database (www.lipidmaps.org) and the Human Metabolome Database (https://hmdb.ca/). Data tables were obtained from the Progenesis QI software and the absolute intensities of all identified compounds were recalculated based on the relative abundance of lipid molecules. The data was initially processed by unsupervised principal component analysis (PCA) to obtain group clusters. Then, the supervised orthogonal partial least-least squares discriminate analysis (OPLS-DA) model was utilized to screen those $\mathrm{m} / \mathrm{z}$ that impacts on the group clustering. All $\mathrm{m} / \mathrm{z}$ with variable importance (VIP) value above 1 were screened for further analysis of variance (ANOVA) using Progenesis QI software. The corresponding lipids of $\mathrm{m} / \mathrm{z}$ with VIP $>1$ and $p$ values $<0.05$ were identified as significant difference. Lastly, pathway enrichment analysis of significantly altered lipids was performed in MetaboAnalyst 4.0. For normality, log transformations and automatic scaling were applied to the data.

\subsection{Primary neuron cultures}

The primary striatal neurons were obtained from mouse embryos (E16 to E17) (Fath et al. 2009; Wu et al. 2016). In brief, corpus striatum was carefully cut into small pieces and subsequently digested with $0.25 \%$ trypsin at $37^{\circ} \mathrm{C}$ for $20 \mathrm{~min}$. The purified neuronal pellet was resuspended with culture medium containing neurobasal medium, 1\% B27 supplement (Gibco), 2 mM Glutamine and 0.2\% Primocin (InvivoGen). Neurons were cultured in 6-well plate with poly-D-ornithine $(0.5 \mathrm{mg} / \mathrm{ml})$-coated coverslip at $37^{\circ} \mathrm{C}$ and $5 \%$ $\mathrm{CO}_{2}$ in a humidified incubator. Half of medium was changed with fresh culture medium every 3 days.

\subsection{Imaging}

Briefly, neurons grown on the coverslip were directly fixed with warm $4 \%$ paraformaldehyde/ $4 \%$ sucrose solution for $10 \mathrm{~min}$ at room temperature after treatment with or without cefepime for $24 \mathrm{~h}$. Neurons were permeabilized with $0.1 \%$ Triton X-100 in PBS for 10 minutes at room temperature, and subsequently blocked with $5 \%$ bovine serum albumin (BSA). The coverslip was incubated with primary antibody (1:50 $1: 1000)$ at $4^{\circ} \mathrm{C}$ overnight, followed by incubation with Alexa-conjugated secondary antibody $(1: 200)$ for 1 h. All IF images were obtained using a laser scanning confocal microscope (Leica SP8 X, Leica) with built-in LAS X software, using a $63 \times 31.3 \mathrm{NA}$ oil lens. In order to ensure the authenticity of the fluorescence statistics, the laser intensity of each sample group was consistent. The following antibodies were used: TOM20 (11802-1-AP, Proteintech), Alexa fluor 647 Phalloidin (A22287, Invitrogen), SV2 (AB2315387, Developmental Studies Hybridoma Bank).

For the quantification of mitochondrial morphology and density, as well as the density of synaptic vesicles, around 20 neurons were blindly selected and utilized for quantification. The quantification 
procedure was performed in Image (Version 1.52P). The line tool was firstly used to trace the profile of neurite, and the size of area or the fluorescence intensity of TOM20 or SV2 was calculated. For obtaining the density of mitochondria, the fluorescence intensity of mitochondria was divided into the corresponding neuritic area.

\subsection{Statistical Analysis}

All data were expressed as mean \pm SD deviation, and measured by GraphPad Prism 8 software using multiple comparison, ordinary one-way Analysis of Variance (ANOVA). The profoundly modified lipids were measured using multiple comparison, two-way ANOVA. The $p$ value $<0.05$ was considered

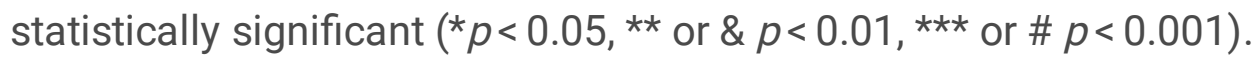

\section{Results}

\subsection{Cefepime administration causes anxiety-like behaviors in mice}

To investigate the effect of cefepime on neurobehaviors, the mice were intraperitoneally administered 80 $\mathrm{mg} / \mathrm{kg}$ cefepime daily for $1,3,5,7$ or 10 days, respectively. Open field test was applied to investigate the effect of cefepime on general activity, exploratory and anxious behavior of mice. Mice were placed into the flat open field box $(48 \times 48 \times 30 \mathrm{~cm})$ after continuous cefepime administration $(80 \mathrm{mg} / \mathrm{kg})$ for indicated time period, and movement path during 5 min of spontaneous movement was simultaneously tracked (Fig. 1a).

Overall, cefepime-treated mice preferred to move around the periphery area of box, especially after cefepime treatment for 5 days. The trajectory around the periphery area displayed an increasing trend in a time-dependent manner (Fig. 1b). In contrast to control mice, the total exploratory time in inner zone of the flat arena gradually decreased with the extension of cefepime administration (Fig. 1b). The total exploratory time spent in inner zone was significantly reduced from the beginning of the fifth day after cefepime treatment (Fig. 1c). The reduced exploratory activity in cefepime-exposed mice was positively correlated to the decreased frequency of entries into the inner zone (Fig. 1d), suggesting that cefepime may induce anxiety-like behavior. Moreover, the total distance traveled in the whole open area was markedly decreased along with the extension of cefepime administration, suggesting that cefepime exposure may impair locomotor activity of mice (Fig. 1e). Collectively, these results showed that treatment of cefepime causes anxiety-like behaviors in mice.

\subsection{Cefepime shows no effect on serum chemistry and organ histology in mice}

Serum chemistry and histology of major organs were assessed to evaluate the toxicity of cefepime on blood profile and the integrity of tissue, respectively. As shown in Fig. 2a, in comparison to the control mice, the levels of ALT, AST and ALP were slightly reduced in the mice treated with cefepime for 5 continuous days. There were no significant difference in the levels of urea, creatinine or uric acid between control and cefepime-treated mice (Fig. 2b). Cefepime showed no obvious effect on the levels of serum lactate dehydrogenase (LDH) and myocardial-associated isoenzyme creatine kinase-MB (CK-MB) 
(Fig. 2c). The levels of serum albumin (ALB) and serum total protein (TP) in cefepime-treated mice were similar to those in control mice (Fig. 2d). Moreover, the concentration of serum cholesterol (CHOL) and triglyceride (TG) as well as the direct bilirubin were not altered by cefepime treatment (Fig. 2e and 2f). Finally, alteration of various organs was microscopically assessed via the histopathology. Cefepime treatment showed no effect on the histology of the major organs, including brain (hippocampus and cerebral cortex), heart, liver, spleen, lung and kidney (Fig. 2g).

\subsection{Cefepime profoundly alters brain lipidomic profile of corpus striatum}

To address the mechanism contributing to behavioral abnormalities in cefepime-treated mice, a UPLCQTOF-MS-based lipidomic approach was applied to characterize the influence on globe lipids among interconnected brain regions, including hippocampus, corpus striatum and cerebral cortex. Firstly, to investigate the differences of lipidomic profile among control group and multiple time-dependent cefepime-treated groups, all identified ion peaks (mass-to-charge ratio, $\mathrm{m} / \mathrm{z}$ ) under both positive ion mode $(E S I+)$ and negative ion mode (ESI-) were subjected to the supervised orthogonal partial least squares discriminant analysis (OPLS-DA) model. The OPLS-DA score plots using one orthogonal component displayed clearly separation under both positive and negative modes among all groups in all three brain regions (Fig. 3a). Notably, with the prolongation of cefepime treatment, the clustering between control and cefepime groups exhibited more manifest in all detected brain regions (Fig. 3a), suggesting that brain lipids were significantly perturbated in cefepime-treated mice. Then, the ion peaks mediating clustering in score plots were investigated and exhibited via loading plot. The value of variable importance (VIP) above $1(\mathrm{VIP} \geq 1)$ was labeled with red color for great contribution to the separation among control and multiple cefepime groups (Fig. 3b). In the three brain regions, corpus striatum, hippocampus and cerebral cortex, 81,111 , and 74 positive ion peaks, and 89,73 and 31 negative ion peaks, respectively, were remarkably modified by cefepime.

Among all detected $\mathrm{m} / \mathrm{z}$ ratios in both modes, the profoundly up-regulated $(p<0.05$, Fold-change $>2)$ and down-regulated $(p<0.05$, Fold-change $<-2) \mathrm{m} / \mathrm{z}$ ratios were subsequently identified. The results discovered much more $\mathrm{m} / \mathrm{z}$ ratios were dysregulated by cefepime administration in corpus striatum than those in hippocampus and cerebral cortex (Fig. 3c). In combination with total numbers satisfying VIP $\geq 1$ under both ESI + and ESI-, corpus striatum was the brain region mostly affected by cefepime (Fig. 3b and 3c).

To determine whether those dysregulated ion peaks in corpus striatum accumulated with the extension of cefepime application, the total number of profoundly altered ion peaks were further separately quantified using the acquired ion peaks from the different time period of cefepime treatment. The results showed that total numbers of $\mathrm{m} / \mathrm{z}$ ratios indeed increased with the extension of cefepime treatment. Notably, the total number of dysregulated $\mathrm{m} / \mathrm{z}$ ratio was significantly increased after cefepime exposure for 5 continuous days ( 149 of $\mathrm{m} / \mathrm{z}$ ratios) than that on day 1 ( 16 of $\mathrm{m} / \mathrm{z}$ ratios) and day 3 ( $56 \mathrm{of} \mathrm{m} / \mathrm{z}$ ratios). Interestingly, there was no obvious changes of dysregulated lipid peaks after cefepime treatment for 7 or 10 days in comparison to cefepime treatment for 5 days continuously. The amount of disturbed lipids 
peaks boosted almost three times from 3 days to 5 days of cefepime treatment, indicating that lipidomic profile of corpus striatum may be remarkably perturbed after cefepime treatment for 5 days (Fig. 3d).

\subsection{Cefepime causes a remarkable dysregulation of glycerophospholipids in corpus striatum}

To discover the differentially changed lipids in the corpus striatum of cefepime-treated mice, those ion peaks with both VIP $\geq 1$ basing on OPLS-DA model and $p<0.05$ acquiring from the statistical analysis were subsequently defined referring to the Lipid Maps Database (www. lipidmaps.org) and the Human Metabolome Database (http://www. hmdb.ca/). The results revealed that a total of 116 lipids of corpus striatum were significantly altered by cefepime (Supplemental Table 1). Basing on the current lipid category, besides a small proportion of lipids without classification, we found that cefepime widely modified seven groups of lipid classes among the total eight categories of lipids (Fig. 4a). The proportion of identified lipid classes glycerophospholipids (GP), glycerolipids (GL), prenol lipids (PR), fatty acyls (FA), sphingolipids (SP), sterol lipids (ST), saccharolipids (SL) and the other uncategorized lipids were $62.07 \%$, $8.62 \%, 6.03 \%, 5.17 \%, 5.17 \%, 3.45 \%, 0.86 \%$ and $8.62 \%$, respectively. Moreover, among all identified glycerophospholipids subclasses, phosphatidylethanolamine (PE) and phosphatidylcholine (PC) were two major subclasses accounting for the most proportion of modified glycerophospholipids caused by cefepime (Fig. 4b). Collectively, cefepime predominantly dysregulated glycerophospholipid profile, especially PC and PE, in the corpus striatum.

Next, the level of change of those modified lipids along with the prolongation of cefepime treatment was quantified and presented in heatmap (Fig. 4c). Interestingly, the majority of PC and PE presented an upregulated trend. In consistent with the discovery in OPLS-DA model, a great number of lipids were remarkably boosted only after continuous treatment with cefepime for 5 days. Those lipids with acute dysregulation were subsequently maintained a relatively stable level after cefepime treatment for 7 or 10 days (Fig. 4c). Lysophosphatidylcholines (LysoPC) was declined at the beginning of cefepime treatment but recovered to normal level from the 5th day. Different from PC and PE, the profile of several other glycerophospholipids subclasses phosphatidylglycerol (PG), phosphatidylinositol (PI) and diacylglycerol (DG) were declined in response to cefepime. Moreover, the lipids of phosphatidylserine (PS), a subclass of GP, were nonuniformly affected in response to cefepime (Fig. 4c). Similar to the GP, most of the lipids belonging to the FA, GL, ST, PR and SP groups were remarkably altered after continuous cefepime treatment for 5 days (Fig. 4d). These data further confirmed that cefepime significantly disturbed the lipid profile after 5 days' treatment. Taken together, cefepime markedly alters lipid profile of corpus striatum, particular PC and PE, which contributes to a large proportion of aberrant lipidomic profile.

\subsection{Cefepime remarkably modifies the composition and structure of glycerophospholipid in corpus striatum}

The effect of cefepime on the composition and structure of glycerophospholipid was studied. The results showed the overall abundance of GP subclasses PE, PC, PS, PG and PI were gradually increased with the extension of cefepime treatment, and maintained relatively stable level after 5 days of cefepime treatment (Fig. 5a). Individually, except for 10 days of cefepime treatment, the gently upregulated 
abundance of PE, PG and PI showed no significance among the control and cefepime-treated groups. Different from these subclasses, cefepime dramatically drove up the abundance of PC and PS after cefepime treatment for 5 days (Fig. 5a).

The major and minor species of PE, PC, PS, PG and PI were separately analyzed. Although the total abundance of $\mathrm{PE}$ only remarkably changed after cefepime treatment for 10 days, the composition of three major PE species (20:5/P-18:1, 20:5/20:0 and 20:4/18:0) were remarkably up-regulated even after cefepime treatment for one day (Fig. 5b). In addition, the composition of another one major PE species (22:6/16:0) and three minor (14:1/24:0, 20:5/18:3 and 0-14:0/18:0) PE species were dramatically increased from the beginning of cefepime treatment for 5 days (Fig. $5 \mathrm{~b}$ and Fig. S1B). Compared to the other GP subclasses, almost all identified major PC species were remarkably up-regulated in response to cefepime treatment on day 5. As shown in the Fig. 5b, six of seventh major PC species were altered by cefepime. Moreover, all identified minor PC species and PS species were also increased by cefepime starting at day 5 (Fig. S1B). Moreover, the composition of two major (20:3/21:0, 22:6/18:0) PS species were also significantly up-regulated by cefepime starting from the 5th day of exposure (Fig. S1A). Although the major species of PG was less affected by cefepime, the minor species of PG (20:0/20:3) was greatly increased after cefepime treatment for 5 days (Fig. S1B). Collectively, cefepime administration largely affected the composition of PC in corpus striatum.

The length and unsaturation degree of acyl chain are two key factors affecting the lipid geometry and fluidity of lipid bilayer, and the distribution of acyl chains differs from disease mutations or depletion of metabolic enzymes (Andresen et al. 1999; Nochi et al. 2017). We then analyzed the pool of fatty acyl chains related to GP. Cefepime treatment uniformly altered the composition of GP, especially from the 5th day of cefepime treatment (Fig. 5c). In addition, the intensity of long fatty acyl chain, such as C32, C34, C36, C37, C38 and C40, were significantly higher in cefepime-treated mice those in control mice (Fig. 5d). Except for the length of acyl chain, the unsaturation degree of acyl chain was also profoundly affected by cefepime (Fig. 5e). Collectively, cefepime significantly remodeled GP profile, especially PC species, accounting for the majority of those modified lipids in response to cefepime treatment.

\subsection{Cefepime causes aberrant neuronal morphology and function in vitro}

To examine the effect of cefepime exposure on lipid disorder of neurons, the embryonic primary neuron isolated from mouse striatum was cultured and treated with cefepime in vitro (DIV). We observed that 50 $\mu \mathrm{M}$ or $500 \mu \mathrm{M}$ cefepime exposure for $24 \mathrm{~h}$ caused a significant increase of fragmented neurotic structure (Fig. 6a). High concentration of cefepime caused more neurite fragmentation; however, the integrity of soma was unaffected and no obvious alteration was noted in cefepime-exposed neurons (Fig. 6a), suggesting cefepime may mainly affect the dendrite of striatal neuron.

Studies have shown that abnormally high or low ratio of PC/PE is able to influence energy metabolism, linking to disease progression (Green et al. 2009; Ledesma et al. 2012). In forementioned results, we quantified the abundance of all PC and PE species, and found that the ratio of PC/PE abundance was significantly increased in vivo from the 7th day of cefepime exposure as compared with control groups 
(Fig. 6b). To further determine whether the increased PC/PE ratio in cefepime-treated brain linked to aberrant energetic system or those abnormal neurites observed in vitro, the morphology of the mitochondria which is the predominate regulator of bioenergetic metabolism was subsequently assessed (Friedman and Nunnari, 2014; Liesa and Shirihai, 2013; Rolfe and Brown, 1997). Mitochondrial morphology of primary striatal neuron was visualized by co-immunostaining with the translocase of mitochondrial outer membrane 20 (TOM20). The results showed that mitochondrial morphology of neuron was obviously altered after cefepime treatment ( $50 \mu \mathrm{M}$ and $500 \mu \mathrm{M})$ for $24 \mathrm{~h}$. In contrast to tubular mitochondrial structure in normal primary neurons, cefepime exposure resulted in swelled and fragmented mitochondria (Fig. 6c). We quantified the area parameter of individual mitochondria using Image $\mathrm{J}$, and found that the mitochondrial size was markedly decreased by cefepime (500 $\mu \mathrm{M})$. In spite that the mitochondrial size showed relatively normal under $50 \mu \mathrm{M}$ cefepime treatment, the overall mitochondrial size was clearly reduced (Fig. 6d). In addition, we further analyzed the average mitochondrial intensity in the selected neuritic area, which was firstly drawn out based on the intensity of the fluorescently-labeled phalloidin, a probe of actin cytoskeleton. Mitochondrial intensity per neuritic area was significantly decreased in cefepime-exposed neurons (Fig. 6e). Taken together, the aberrant of mitochondrial morphology and the diminished mitochondrial content in neurite may be associated with those abnormal neurites observed in cefepime-treated striatal neuron in vitro.

Through immunostaining analysis of synaptic vesicle glycoprotein 2 (SV2), a key secretory vesicle protein in neurons (Ahnert-Hilger et al. 2013; M.-K. Chen et al. 2018; Stout et al. 2019), we evaluated the function alteration in the cultured striatal neuron in response to cefepime treatment. We found that the density of synaptic vesicle was obviously reduced by cefepime treatment (Fig. $6 \mathrm{~g}$ and $6 \mathrm{f}$ ), implying that cefepime treatment impaired the formation of synaptic vesicles in neurites. Taken together, these results indicated that cefepime treatment may interfere with neurotic morphology, axonal mitochondria and synaptic vesicle, which may eventually lead to the deficiency of neuronal function.

\section{Discussion}

The mechanism for the CNS adverse effect of cefepime has not been fully elucidated. Using lipidomic analysis, we aimed to investigate whether the disorder of lipids hemostasis participates such adverse effect. We observed that 5-day continuous intraperitoneal injection of cefepime dysregulated the hemostasis of glycerophospholipid profile in the corpus striatum of mice. Cefepime not only dysregulated the abundance of GP but also altered biophysical properties of neuronal membrane through modifying the length and unsaturation of acyl chain. Morphological analysis of primary striatal neuron revealed that cefepime-induced GP disorder may contribute to the altered neuritic integrity and mitochondrial dynamic process, and eventually lead to the reduction of synaptic vesicles in neuron. Similar to previous studies, cefepime dysregulated lipids profile in the striatum corpus, an area accepting all excitatory glutamatergic neuron from cortical neuron (Mandelbaum et al. 2019). Collectively, our data show that cefepime causes disorder of GP in the striatum corpus, which may underlie the CNS adverse effect of cefepime. 


\subsection{Comprehensive lipidomic analysis reveals a mechanism underlying the CNS adverse effect of cefepime}

CNS adverse effect of cefepime ranges from $10 \sim 23 \%$, and often occurs in the elder patients (Drago and Vecchi, 2008; Ojha et al. 2020; Payne et al. 2017). Monotherapy with cefepime in clinic is usually administered intravenously $2 \sim 4 \mathrm{~g} /$ day (33 $66 \mathrm{mg} / \mathrm{kg} /$ day for human dose), and the trough level of cefepime arrives to $11.5 \sim 44.6 \mathrm{mg} / \mathrm{L}$. Previous studies showed that the trough level of cefepime above $20 \mathrm{mg} / \mathrm{L}$ has a $50 \%$ probability of developing neurological symptom (Lamoth et al. 2010). In this study, to avoid general toxicity of mice which may interfere with the lipidomic profiling of brain, mice were injected daily with $80 \mathrm{mg} / \mathrm{kg}$ cefepime, which is half of equivalent dose in human based on body surface area (Nair and Jacob, 2016). Similar to patients in clinic, the anxiety-like behavior as well as the attenuated locomotor activity were observed in the mice treated with cefepime for 5 continuous days. Both serum chemistry and histopathology of the major organs showed no significant alteration.

The pathophysiology of cefepime neurotoxicity is thought to across BBB and modulate GABA receptor. As the brain is the second most lipid-rich organ, we hypothesized that dysregulated lipid metabolism may participate in the initial or developing progress of cefepime neurotoxicity. To this end, through UPLCMS/MS based lipidomic analysis, we found a widely alteration of lipid profile in all three interconnected brain areas. In particular, the number of dysregulated lipids were remarkedly increased after 5-days of cefepime exposure, supporting the findings in the neurobehaviors of cefepime-treated mice.

It is well known that homeostasis of lipids as well as fatty acids are the crucial determinants of neural development, neurotransmitter releasing, and receptor function of neurons (Dorninger et al. 2019; Gantz and Bean, 2017; Josey et al. 2020). Dyshomeostasis of lipids or fatty acids in neurons has been found to mediate CNS disorders. For instance, the disruption of PC homeostasis mediates a series of neuropathies (Kim et al. 2018; Yu-Yuan and Yi-Jun, 2020), and accumulation of arachidonic acid-containing PC species in the hypothalamus and striatum mediates the depressive behavior in rats (Green et al. 2009). Therefore, we propose that the dysregulated lipids may contribute to abnormal neurobehaviors caused by cefepime in mice.

\subsection{Cefepime predominately dysregulates glycerophospholipid profile of corpus striatum}

Through combined OPLS-DA model and statistical analysis, we discovered that the ratio of dysregulated ion peaks of corpus striatum was higher than that in hippocampus or prefrontal cortex, indicating that dysregulated lipids of corpus striatum may contribute to cefepime-induced CNS disorders. Cefepime is able to competes with $y$-aminobutyric acid, thus weakening the function of GABAergic neuron and eventually leading to CNS disorder (Lam and Gomolin, 2006). In addition, the proportion and diversity of GABAergic neuron within the striatum are higher than that in the cortex or hippocampus (Huang and Paul, 2019; Murata and Colonnese, 2020; Shi et al. 2021). Thinking above, we speculate that GABAergic neuron-enriched striatum may be more sensitive to cefepime treatment than the other two brain areas. Indeed, the data from lipidomic profile revealed that corpus striatum was the brain region that was mostly affected by cefepime. 
Our results showed that GP accounted for the majority of cefepime-dysregulated lipids. GP mainly act as amphipathic molecules for forming the backbone of biological membrane as well as a reservoir for the generation of multiple bioactive mediators. Moreover, GP serve as the precursors for the synthesis of diacylglycerol which favors membrane fusion, participates in neurotransmitter release, and modulate the activities of diverse kinases, such as 5-lipoxygenase (Hishikawa et al. 2017; Kumar et al. 2020; Ziegler and Tavosanis, 2019). Cefepime treatment resulted in the abnormalities in the neurite, mitochondria and synaptic vesicle, which are the biological membrane-enriched structure in neurons. We speculate that the identified aberrant GP in striatum may contribute to, at least in part, the aberrant membrane structure, which eventually leads to the abnormal neurobehaviors. Except for the composition of lipid, the length and unsaturation degree of acyl chain are another two key parameters in determining the biological membrane order, packing pattern, surface charge and fluidity (Fidalgo Rodríguez et al. 2019). We noticed that cefepime also caused dysregulated length and unsaturation degree of acyl chain. More studies are required to identify whether aberrant GP profile in corpus striatum disrupts membrane structure in the future.

\subsection{Cefepime-dysregulated lipid profile may result in abnormal morphology of striatal neuron}

It has been known that lipid dyshomeostasis in driving abnormal neurological behavior differs a lot (Chew et al. 2020; Krishna et al. 2016). For instance, the dyshomeostasis of phospholipid phosphatidylinositol-4,5-bisphosphate (Ptdlns $\left.(4,5) \mathrm{P}_{2}\right)$ may contribute to brain dysfunction and cognitive disabilities in Down's syndrome (Voronov et al. 2008). A decline in the level of omega-3 fatty acids leads to lipid peroxidation, resulting in neuronal degeneration and aging (J. Chen et al. 2017). In this study, cefepime treatment clearly altered the morphology of lipid biolayer-enriched neurite, exhibiting a fragmented dot-like structure; however, nucleus membrane was unaffected. As the highly branched neurites account for the majority of lipid bilayer structure of neurons, our findings support a notion that maintenance of neuritic structure depends on the hemostasis of lipids, especially on GP, the ubiquitous building blocks of biological membrane (Araki and Wurtman, 1997; Kuge et al. 2014; Paoletti et al. 2011). We propose that the dysregulated GP in the striatal region of cefepime-treated mice may impair the integrity of neurites, eventually leading to the neuronal dysfunction.

$\mathrm{PC}$ and $\mathrm{PE}$ comprise the most two subclasses of GP, and irregular high or low ratio of PC/PE is able to perturb energy metabolism correlating to mitochondria (Basu Ball et al. 2018; Chabi et al. 2018; Rodríguez-Arribas et al. 2017; van der Veen et al. 2017). Our study showed that cefepime treatment profoundly up-regulated the ratio of PC/PE species in the striatum. Morphology analysis visualized an altered mitochondrial morphology from tubules to swelled fragments. Moreover, the accumulation of arachidonic acid, the side product of GP catabolism, produces a variety of detrimental effects on neural membrane, including severe adverse effect on mitochondria (Angelova et al. 2021; Chabi et al. 2018). Consistently, the mitochondrial structure and mitochondrial density in the cultured striatal neuron was profoundly reduced after cefepime treatment. We infer that the aberrant mitochondrial morphology and reduced mitochondrial content in neurite may participate neurite degeneration. Interestingly, we also observed a decreased density of synaptic vesicle in the neurite. Due to the essential role of synaptic 
vesicle in neurotransmission (Brunger et al. 2018; Südhof, 2013), the reduced synaptic vesicles may somehow represent the reduction of neuronal activity.

In summary, cefepime profoundly changes the GP profile in the corpus striatum of mice. Cefepime alters the membrane structure of neurites, mitochondria and synaptic vesicle in the cultured striatal neurons. Our study reveals that dysregulation of GP homeostasis may contribute to cefepime-induced CNS adverse effect.

\section{Declarations}

\section{Ethical approval}

All applicable international, national and/or institutional guidelines for the care and use of animals were followed. All experimental procedures and use of the animals were conducted in accordance with the guidelines established by the Association for Assessment and Accreditation of Laboratory Animal Care and the Institutional Animal Care and Use Committee of Sichuan University. All efforts were made to minimize the suffering of the mice.

\section{Conflict of Interest}

The authors declare that they have no conflicts of interest. The authors have no financial or proprietary interests in any material discussed in this article.

\section{Consent to participate}

Not applicable

\section{Consent for publication:}

Not applicable

\section{Funding}

This work was partially supported by National Natural Science Foundation of China (Grants 32000719 , 82071494, 81871043, 81272459), the National Science and Technology Major Project (2018ZX09201017-009), and "1·3.5 Project for Disciplines of Excellence, West China Hospital, Sichuan University".

\section{Author Contributions}

X.B.C, X.C.L and X.J.W conceived and designed the experiments. X.C.L and X.J.W performed the experiments and analyzed the data. R.X, S.M.W, H.L.Z, J.M.Z, X.M.W, Q.F.W, X.W.Y, Y.Y.C, L.H.J, Y.M.H, S.L, R.C, Y.H.W, Y.X.C, F.Q, Y.P.D, H.C.L, Y.Z, H.Q.Z, Q.B, H.B.W, J.W.T and Y.L.Z contributed reagents, materials, analysis tools and helped some experiments. X.B.C and L.W wrote and revised the main manuscript text. All authors read and approved the final manuscript. 


\section{Acknowledgments}

We would like to thank Ms.Liang Wang for help with guidance on experimental techniques and writing.

\section{References}

1. Ahnert-Hilger G, Münster-Wandowski A, Höltje M. Synaptic vesicle proteins: targets and routes for botulinum neurotoxins. Curr Top Microbiol Immunol. 2013;364:159-77. https://doi.org/10.1007/9783-642-33570-9_8.

2. Amakhin DV, Soboleva EB, Zaitsev AV. Cephalosporin antibiotics are weak blockers of GABAa receptor-mediated synaptic transmission in rat brain slices. Biochem Biophys Res Commun. 2018;499(4):868-74. https://doi.org/10.1016/j.bbrc.2018.04.008.

3. Andresen BS, Olpin S, Poorthuis BJHM, Scholte HR, Vianey-Saban C, Wanders R, ljlst L, Morris A, Pourfarzam M, Bartlett K, Baumgartner ER, DeKlerk JBC, Schroeder LD, Corydon TJ, Lund H, Winter V, Bross P, Bolund L, Gregersen N. Clear Correlation of Genotype with Disease Phenotype in Very-LongChain Acyl-CoA Dehydrogenase Deficiency. The American Journal of Human Genetics. 1999;64(2):479-94. https://doi.org/10.1086/302261.

4. Angelova PR, Esteras N, Abramov AY. Mitochondria and lipid peroxidation in the mechanism of neurodegeneration: Finding ways for prevention. Med Res Rev. 2021;41(2):770-84. https://doi.org/10.1002/med.21712.

5. Araki W, Wurtman RJ. (1997). Control of membrane phosphatidylcholine biosynthesis by diacylglycerol levels in neuronal cells undergoing neurite outgrowth. Proceedings of the National Academy of Sciences, 94(22), 11946-11950. https://doi.org/10.1073/pnas.94.22.11946.

6. Basu Ball W, Neff JK, Gohil VM. The role of nonbilayer phospholipids in mitochondrial structure and function. FEBS Lett. 2018;592(8):1273-90. https://doi.org/10.1002/1873-3468.12887.

7. Beaulieu C, Kurczewski L, Yajnik V. Cefepime challenge after piperacillin/tazobactam-induced thrombocytopenia. J Thromb Thrombolysis. 2019;48(1):167-70. https://doi.org/10.1007/s11239019-01848-3.

8. Bhagwat SS, Periasamy H, Takalkar SS, Palwe SR, Khande HN, Patel MV. (2019). The Novel $\beta-$ Lactam Enhancer Zidebactam Augments the In Vivo Pharmacodynamic Activity of Cefepime in a Neutropenic Mouse Lung Acinetobacter baumannii Infection Model. Antimicrob Agents Chemother, 63(4). https://doi.org/10.1128/AAC.02146-18.

9. Bhattacharyya S, Darby RR, Raibagkar P, Gonzalez Castro LN, Berkowitz AL. Antibiotic-associated encephalopathy. Neurology. 2016;86(10):963-71. https://doi.org/10.1212/WNL.0000000000002455.

10. Brunger AT, Choi UB, Lai Y, Leitz J, Zhou Q. Molecular Mechanisms of Fast Neurotransmitter Release. Annual Review of Biophysics. 2018;47(1):469-97. https://doi.org/10.1146/annurev-biophys-070816034117. 
11. Castro JP, Jung T, Grune T, Siems W. 4-Hydroxynonenal (HNE) modified proteins in metabolic diseases. Free Radic Biol Med. 2017;111:309-15. https://doi.org/10.1016/j.freeradbiomed.2016.10.497.

12. Chabi B, Fouret G, Lecomte J, Cortade F, Pessemesse L, Baati N, Coudray C, Lin L, Tong Q, WrutniakCabello C, Casas F, Feillet-Coudray C. Skeletal muscle overexpression of short isoform Sirt3 altered mitochondrial cardiolipin content and fatty acid composition. J Bioenerg Biomembr. 2018;50(2):131-42. https://doi.org/10.1007/s10863-018-9752-1.

13. Chen J, Wei Y, Chen X, Jiao J, Zhang Y. Polyunsaturated fatty acids ameliorate aging via redoxtelomere-antioncogene axis. Oncotarget. 2017;8(5):7301-14.

https://doi.org/10.18632/oncotarget.14236.

14. Chen M-K, Mecca AP, Naganawa M, Finnema SJ, Toyonaga T, Lin S, Najafzadeh S, Ropchan J, Lu Y, McDonald JW, Michalak HR, Nabulsi NB, Arnsten AFT, Huang Y, Carson RE, van Dyck CH. Assessing Synaptic Density in Alzheimer Disease With Synaptic Vesicle Glycoprotein 2A Positron Emission Tomographic Imaging. JAMA Neurology. 2018;75(10):1215. https://doi.org/10.1001/jamaneurol.2018.1836.

15. Chew H, Solomon VA, Fonteh AN. Involvement of Lipids in Alzheimer's Disease Pathology and Potential Therapies. Front Physiol. 2020;11(June):1-28. https://doi.org/10.3389/fphys.2020.00598.

16. Díaz M, Fabelo N, Casañas-Sánchez V, Marin R, Gómez T, Quinto-Alemany D, Pérez JA. Hippocampal Lipid Homeostasis in APP/PS1 Mice is Modulated by a Complex Interplay Between Dietary DHA and Estrogens: Relevance for Alzheimer's Disease. Journal of Alzheimer's Disease. 2015;49(2):459-81. https://doi.org/10.3233/JAD-150470.

17. Diaz M, Retamal MA. (2018). Editorial: Modulation of Ion Channels and lonic Pumps by Fatty Acids: Implications in Physiology and Pathology. Frontiers in Physiology, 9. https://doi.org/10.3389/fphys.2018.01625.

18. Dorninger F, König T, Scholze P, Berger ML, Zeitler G, Wiesinger C, Gundacker A, Pollak DD, Huck S, Just WW, Forss-Petter S, Pifl C, Berger J. Disturbed neurotransmitter homeostasis in ether lipid deficiency. Hum Mol Genet. 2019;28(12):2046-61. https://doi.org/10.1093/hmg/ddz040.

19. Drago L, Vecchi E, De. The safety of cefepime in the treatment of infection. Expert Opinion on Drug Safety. 2008;7(4):377-87. https://doi.org/10.1517/14740338.7.4.377.

20. Fang F, Zhan Y, Hammar N, Shen X, Wirdefeldt K, Walldius G, Mariosa D. Lipids, Apolipoproteins, and the Risk of Parkinson Disease. Circ Res. 2019;125(6):643-52. https://doi.org/10.1161/CIRCRESAHA.119.314929.

21. Fanning S, Haque A, Imberdis T, Baru V, Barrasa MI, Nuber S, Termine D, Ramalingam N, Ho GPH, Noble T, Sandoe J, Lou Y, Landgraf D, Freyzon Y, Newby G, Soldner F, Terry-Kantor E, Kim T-E, Hofbauer HF, ... Selkoe D. Lipidomic Analysis of a-Synuclein Neurotoxicity Identifies Stearoyl CoA Desaturase as a Target for Parkinson Treatment. Mol Cell. 2019;73(5):1001-14.e8. https://doi.org/10.1016/j.molcel.2018.11.028. 
22. Fath T, Ke YD, Gunning P, Götz J, Ittner LM. Primary support cultures of hippocampal and substantia nigra neurons. Nat Protoc. 2009;4(1):78-85. https://doi.org/10.1038/nprot.2008.199.

23. Fecchio C, Palazzi L, de Laureto PP. a-Synuclein and Polyunsaturated Fatty Acids: Molecular Basis of the Interaction and Implication in Neurodegeneration. Molecules. 2018;23(7):1531. https://doi.org/10.3390/molecules23071531.

24. Fidalgo Rodríguez JL, Dynarowicz-Latka P, Minones Conde J. Interactions of cholesterol and 7ketocholesterol with unsaturated fatty acids of different unsaturation degree - The monolayer study. Biochimica et Biophysica Acta (BBA) - Biomembranes. 2019;1861(8):1428-36. https://doi.org/10.1016/j.bbamem.2019.06.002.

25. Friedman JR, Nunnari J. Mitochondrial form and function. Nature. 2014;505(7483):335-43. https://doi.org/10.1038/nature12985.

26. Gantz SC, Bean BP. Cell-Autonomous Excitation of Midbrain Dopamine Neurons by Endocannabinoid-Dependent Lipid Signaling. Neuron. 2017;93(6):1375-87.e2. https://doi.org/10.1016/j.neuron.2017.02.025.

27. Green P, Anyakoha N, Yadid G, Gispan-Herman I, Nicolaou A. Arachidonic acid-containing phosphatidylcholine species are increased in selected brain regions of a depressive animal model: Implications for pathophysiology. Prostaglandins Leukot Essent Fatty Acids. 2009;80(4):213-20. https://doi.org/10.1016/j.plefa.2009.02.005.

28. Hanada K. Lipid transfer proteins rectify inter-organelle flux and accurately deliver lipids at membrane contact sites. J Lipid Res. 2018;59(8):1341-66. https://doi.org/10.1194/jlr.R085324.

29. Hishikawa D, Valentine WJ, lizuka-Hishikawa Y, Shindou H, Shimizu T. Metabolism and functions of docosahexaenoic acid-containing membrane glycerophospholipids. FEBS Lett. 2017;591(18):273044. https://doi.org/10.1002/1873-3468.12825.

30. Honore PM, Spapen HD. Cefepime-induced neurotoxicity in critically ill patients undergoing continuous renal replacement therapy: beware of dose reduction! Crit Care. 2015;19(1):455. https://doi.org/10.1186/s13054-015-1179-z.

31. Huang ZJ, Paul A. The diversity of GABAergic neurons and neural communication elements. Nat Rev Neurosci. 2019;20(9):563-72. https://doi.org/10.1038/s41583-019-0195-4.

32. Imberdis T, Negri J, Ramalingam N, Terry-Kantor E, Ho GPH, Fanning S, Stirtz G, Kim T-E, Levy OA, Young-Pearse TL, Selkoe D, Dettmer U. (2019). Cell models of lipid-rich a-synuclein aggregation validate known modifiers of a-synuclein biology and identify stearoyl-CoA desaturase. Proceedings of the National Academy of Sciences, 116(41), 20760-20769. https://doi.org/10.1073/pnas.1903216116.

33. loannou MS, Jackson J, Sheu S-H, Chang C-L, Weigel AV, Liu H, Pasolli HA, Xu CS, Pang S, Matthies D, Hess HF, Lippincott-Schwartz J, Liu Z. Neuron-Astrocyte Metabolic Coupling Protects against Activity-Induced Fatty Acid Toxicity. Cell. 2019;177(6):1522-35.e14. https://doi.org/10.1016/j.cell.2019.04.001. 
34. Josey BP, Heinrich F, Silin V, Lösche M. Association of Model Neurotransmitters with Lipid Bilayer Membranes. Biophys J. 2020;118(5):1044-57. https://doi.org/10.1016/j.bpj.2020.01.016.

35. Kamboj AK, Yung-Lun Chin J, Huebert RC. A Curious Case of Confusion in a Patient With Cirrhosis. Gastroenterology. 2020;159(6):2036-8. https://doi.org/10.1053/j.gastro.2020.04.030.

36. Khasani S. Cefepime-induced jaw myoclonus. Neurology. 2015;84(11):1183-3. https://doi.org/10.1212/WNL.0000000000001365.

37. Kim ST, Kyung EJ, Suh JS, Lee HS, Lee JH, Chae SI, Park ES, Chung YH, Bae J, Lee TJ, Lee WM, Sohn UD, Jeong JH. Phosphatidylcholine attenuated docetaxel-induced peripheral neurotoxicity in rats. Drug Chem Toxicol. 2018;41(4):476-85. https://doi.org/10.1080/01480545.2017.1390580.

38. Krishna S, Lin Z, de La Serre CB, Wagner JJ, Harn DH, Pepples LM, Djani DM, Weber MT, Srivastava L, Filipov NM. Time-dependent behavioral, neurochemical, and metabolic dysregulation in female C57BL/6 mice caused by chronic high-fat diet intake. Physiol Behav. 2016;157:196-208. https://doi.org/10.1016/j.physbeh.2016.02.007.

39. Kuge H, Akahori K, Yagyu K, Honke K. Functional Compartmentalization of the Plasma Membrane of Neurons by a Unique Acyl Chain Composition of Phospholipids. J Biol Chem. 2014;289(39):2678393. https://doi.org/10.1074/jbc.M114.571075.

40. Kumar A, Behl T, Jamwal S, Kaur I, Sood A, Kumar P. Exploring the molecular approach of COX and LOX in Alzheimer's and Parkinson's disorder. Mol Biol Rep. 2020;47(12):9895-912. https://doi.org/10.1007/s11033-020-06033-x.

41. Kunkle BW, Grenier-Boley B, Sims R, Bis JC, Damotte V, Naj AC, Boland A, Vronskaya M, van der Lee SJ, Amlie-Wolf A, Bellenguez C, Frizatti A, Chouraki V, Martin ER, Sleegers K, Badarinarayan N, Jakobsdottir J, Hamilton-Nelson KL, Moreno-Grau S, ... Pericak-Vance MA. Genetic meta-analysis of diagnosed Alzheimer's disease identifies new risk loci and implicates $A \beta$, tau, immunity and lipid processing. Nat Genet. 2019;51(3):414-30. https://doi.org/10.1038/s41588-019-0358-2.

42. Lam S, Gomolin IH. (2006). Cefepime neurotoxicity: Case report, pharmacokinetic considerations, and literature review. In Pharmacotherapy. https://doi.org/10.1592/phco.26.8.1169.

43. Lamoth F, Buclin T, Pascual A, Vora S, Bolay S, Decosterd LA, Calandra T, Marchetti O. High Cefepime Plasma Concentrations and Neurological Toxicity in Febrile Neutropenic Patients with Mild Impairment of Renal Function. Antimicrob Agents Chemother. 2010;54(10):4360-7. https://doi.org/10.1128/AAC.01595-08.

44. Ledesma MD, Martin MG, Dotti CG. Lipid changes in the aged brain: Effect on synaptic function and neuronal survival. Prog Lipid Res. 2012;51(1):23-35. https://doi.org/10.1016/j.plipres.2011.11.004.

45. Liesa M, Shirihai OS. Mitochondrial Dynamics in the Regulation of Nutrient Utilization and Energy Expenditure. Cell Metab. 2013;17(4):491-506. https://doi.org/10.1016/j.cmet.2013.03.002.

46. Mandelbaum G, Taranda J, Haynes TM, Hochbaum DR, Huang KW, Hyun M, Umadevi Venkataraju K, Straub C, Wang W, Robertson K, Osten P, Sabatini BL. Distinct Cortical-Thalamic-Striatal Circuits through the Parafascicular Nucleus. Neuron. 2019;102(3):636-52.e7. https://doi.org/10.1016/j.neuron.2019.02.035. 
47. Manni MM, Tiberti ML, Pagnotta S, Barelli H, Gautier R, Antonny B. (2018). Acyl chain asymmetry and polyunsaturation of brain phospholipids facilitate membrane vesiculation without leakage. ELife, 7 . https://doi.org/10.7554/eLife.34394.

48. Mesa-Herrera, Taoro-González. Valdés-Baizabal, Diaz, and Marín. (2019). Lipid and Lipid Raft Alteration in Aging and Neurodegenerative Diseases: A Window for the Development of New Biomarkers. International Journal of Molecular Sciences, 20(15), 3810. https://doi.org/10.3390/ijms20153810.

49. Murata Y, Colonnese MT. GABAergic interneurons excite neonatal hippocampus in vivo. Science Advances. 2020. https://doi.org/10.1126/sciadv.aba1430.

50. Nair A, Jacob S. A simple practice guide for dose conversion between animals and human. Journal of Basic Clinical Pharmacy. 2016;7(2):27. https://doi.org/10.4103/0976-0105.177703.

51. Nochi Z, Olsen RKJ, Gregersen N. Short-chain acyl-CoA dehydrogenase deficiency: from gene to cell pathology and possible disease mechanisms. J Inherit Metab Dis. 2017;40(5):641-55. https://doi.org/10.1007/s10545-017-0047-1.

52. Ojha N, Riaz S, Eranki A. (2020). Cefepime Neurotoxicity in a Patient With Acute Tubular Necrosis. Cureus. https://doi.org/10.7759/cureus.9911.

53. Paoletti L, Elena C, Domizi P, Banchio C. Role of phosphatidylcholine during neuronal differentiation. IUBMB Life. 2011;63(9):714-20. https://doi.org/10.1002/iub.521.

54. Payne LE, Gagnon DJ, Riker RR, Seder DB, Glisic EK, Morris JG, Fraser GL. Cefepime-induced neurotoxicity: a systematic review. Crit Care. 2017;21(1):276. https://doi.org/10.1186/s13054-0171856-1.

55. Pechère J-C, Vladoianu IR. Development of resistance during ceftazidime and cefepime therapy in a murine peritonitis model. J Antimicrob Chemother. 1992;29(5):563-73. https://doi.org/10.1093/jac/29.5.563.

56. Rao JS, Rapoport SI, Kim H-W. Altered neuroinflammatory, arachidonic acid cascade and synaptic markers in postmortem Alzheimer's disease brain. Translational Psychiatry. 2011;1(8):e31-1. https://doi.org/10.1038/tp.2011.27.

57. Rodríguez-Arribas M, Yakhine-Diop SMS, Pedro JMBB-S, Gómez-Suaga P, Gómez-Sánchez R, Martínez-Chacón G, Fuentes JM, González-Polo RA, Niso-Santano M. Mitochondria-Associated Membranes (MAMs): Overview and Its Role in Parkinson's Disease. Mol Neurobiol. 2017;54(8):6287303. https://doi.org/10.1007/s12035-016-0140-8.

58. Rolfe DFS, Brown GC. Cellular energy utilization and molecular origin of standard metabolic rate in mammals. Physiol Rev. 1997;77(3):731-58. https://doi.org/10.1152/physrev.1997.77.3.731.

59. Serricchio M, Vissa A, Kim PK, Yip CM, McQuibban GA. Cardiolipin synthesizing enzymes form a complex that interacts with cardiolipin-dependent membrane organizing proteins. Biochimica et Biophysica Acta (BBA) - Molecular Cell Biology of Lipids. 2018;1863(4):447-57. https://doi.org/10.1016/j.bbalip.2018.01.007. 
60. Shi M-Y, Ding L-F, Guo Y-H, Cheng Y-X, Bi G-Q, Lau P-M. Long-range GABAergic projections from the nucleus of the solitary tract. Molecular Brain. 2021;14(1):38. https://doi.org/10.1186/s13041-02100751-4.

61. Shirane M, Wada M, Morita K, Hayashi N, Kunimatsu R, Matsumoto Y, Matsuzaki F, Nakatsumi H, Ohta K, Tamura Y, Nakayama KI. Protrudin and PDZD8 contribute to neuronal integrity by promoting lipid extraction required for endosome maturation. Nat Commun. 2020;11(1):4576. https://doi.org/10.1038/s41467-020-18413-9.

62. Stout KA, Dunn AR, Hoffman C, Miller GW. The Synaptic Vesicle Glycoprotein 2: Structure, Function, and Disease Relevance. ACS Chemical Neuroscience. 2019;10(9):3927-38. https://doi.org/10.1021/acschemneuro.9b00351.

63. Südhof TC. Neurotransmitter Release: The Last Millisecond in the Life of a Synaptic Vesicle. Neuron. 2013;80(3):675-90. https://doi.org/10.1016/j.neuron.2013.10.022.

64. Sweeney MD, Sagare AP, Zlokovic BV. Blood-brain barrier breakdown in Alzheimer disease and other neurodegenerative disorders. Nature Reviews Neurology. 2018;14(3):133-50. https://doi.org/10.1038/nrneurol.2017.188.

65. Tomihara K, Soga T, Nomura M, Korach KS, Gustafsson J-Å, Pfaff DW, Ogawa S. Effect of ER- $\beta$ gene disruption on estrogenic regulation of anxiety in female mice. Physiol Behav. 2009;96(2):300-6. https://doi.org/10.1016/j.physbeh.2008.10.014.

66. Valadas JS, Esposito G, Vandekerkhove D, Miskiewicz K, Deaulmerie L, Raitano S, Seibler P, Klein C, Verstreken P. ER Lipid Defects in Neuropeptidergic Neurons Impair Sleep Patterns in Parkinson's Disease. Neuron. 2018;98(6):1155-69.e6. https://doi.org/10.1016/j.neuron.2018.05.022.

67. van der Veen JN, Kennelly JP, Wan S, Vance JE, Vance DE, Jacobs RL. The critical role of phosphatidylcholine and phosphatidylethanolamine metabolism in health and disease. Biochimica et Biophysica Acta (BBA) - Biomembranes. 2017;1859(9):1558-72. https://doi.org/10.1016/j.bbamem.2017.04.006.

68. Voronov SV, Frere SG, Giovedi S, Pollina EA, Borel C, Zhang H, Schmidt C, Akeson EC, Wenk MR, Cimasoni L, Arancio O, Davisson MT, Antonarakis SE, Gardiner K, De Camilli P, Di Paolo G. (2008). Synaptojanin 1-linked phosphoinositide dyshomeostasis and cognitive deficits in mouse models of Down's syndrome. Proceedings of the National Academy of Sciences, 105(27), 9415-9420. https://doi.org/10.1073/pnas.0803756105.

69. Wang B, Tontonoz P. Phospholipid Remodeling in Physiology and Disease. Annu Rev Physiol. 2019;81(1):165-88. https://doi.org/10.1146/annurev-physiol-020518-114444.

70. Wu X, Fleming A, Ricketts T, Pavel M, Virgin H, Menzies FM, Rubinsztein DC. Autophagy regulates Notch degradation and modulates stem cell development and neurogenesis. Nat Commun. 2016;7(1):10533. https://doi.org/10.1038/ncomms10533.

71. Yang C, Wang X, Wang J, Wang X, Chen W, Lu N, Siniossoglou S, Yao Z, Liu K. Rewiring Neuronal Glycerolipid Metabolism Determines the Extent of Axon Regeneration. Neuron. 2020;105(2):27692.e5. https://doi.org/10.1016/j.neuron.2019.10.009. 
72. Yassine HN, Braskie MN, Mack WJ, Castor KJ, Fonteh AN, Schneider LS, Harrington MG, Chui HC. Association of Docosahexaenoic Acid Supplementation With Alzheimer Disease Stage in Apolipoprotein E \&4 Carriers. JAMA Neurology. 2017;74(3):339. https://doi.org/10.1001/jamaneurol.2016.4899.

73. Yu-Yuan L, Yi-Jun W. Changes of Neuropathy Target Esterase Affect Phospholipid Homeostasis in SK-N-SH Cells. Rev Chim. 2020;71(8):327-34. https://doi.org/10.37358/RC.20.8.8306.

74. Ziegler AB, Tavosanis G. Glycerophospholipids - Emerging players in neuronal dendrite branching and outgrowth. Dev Biol. 2019;451(1):25-34. https://doi.org/10.1016/j.ydbio.2018.12.009.

\section{Figures}

A

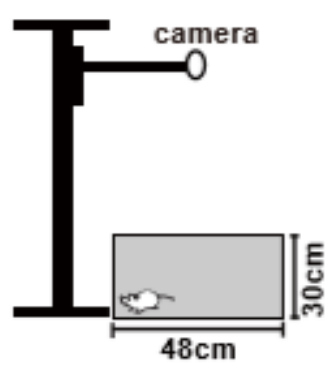

D

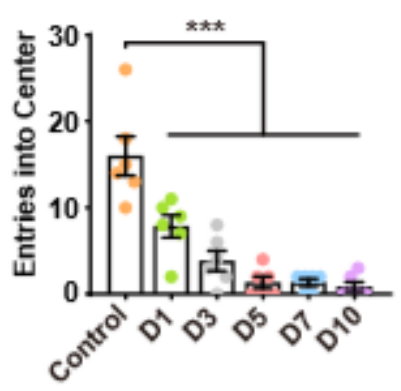

C

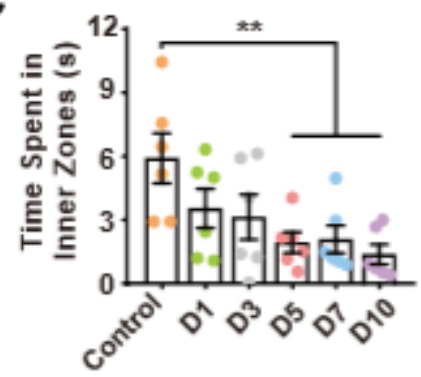

E

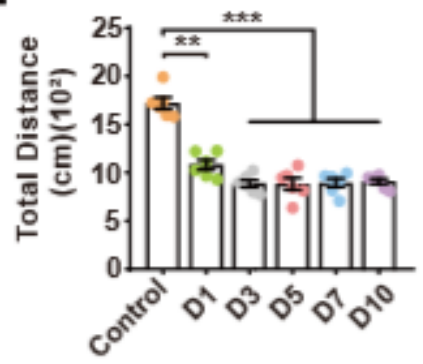

B

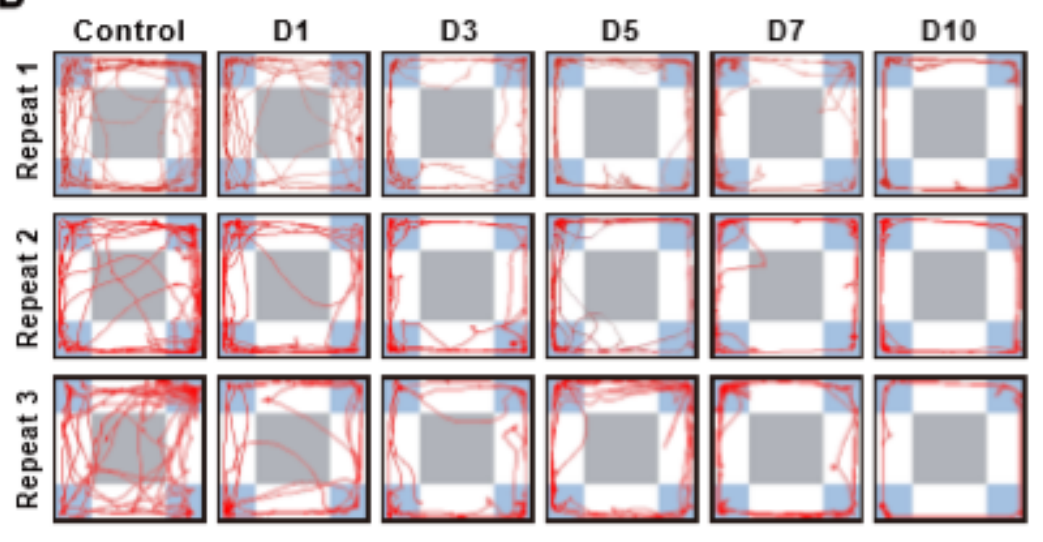

Figure 1

Cefepime causes abnormal neurological behavior. (a) Schematic of experimental illustration on the open field apparatus. (b) The representative trajectory of mouse movement in the open field apparatus. Three trajectory images of control and cefepime-treated group at day 1 (D1), day 3 (D3), day 5 (D5), day 7 (D7) 
and day 10 (D10) are shown, respectively. The trajectory of mouse movement was displayed with red line. (c) Total time (s) spent in the inner zone of open field box during 5 minutes of measurement. (d) Total numbers of entrances into the inner zone during 5 minutes of measurement. (e) Total distance $(\mathrm{cm})$ traveled in the whole open field area during 5 minutes of measurement. For $c, d, e$, data from six mice $(n=6)$ for all groups were used for quantification, One-way ANOVA, $* *, p<0.01 ; * \star *, p<0.001$.

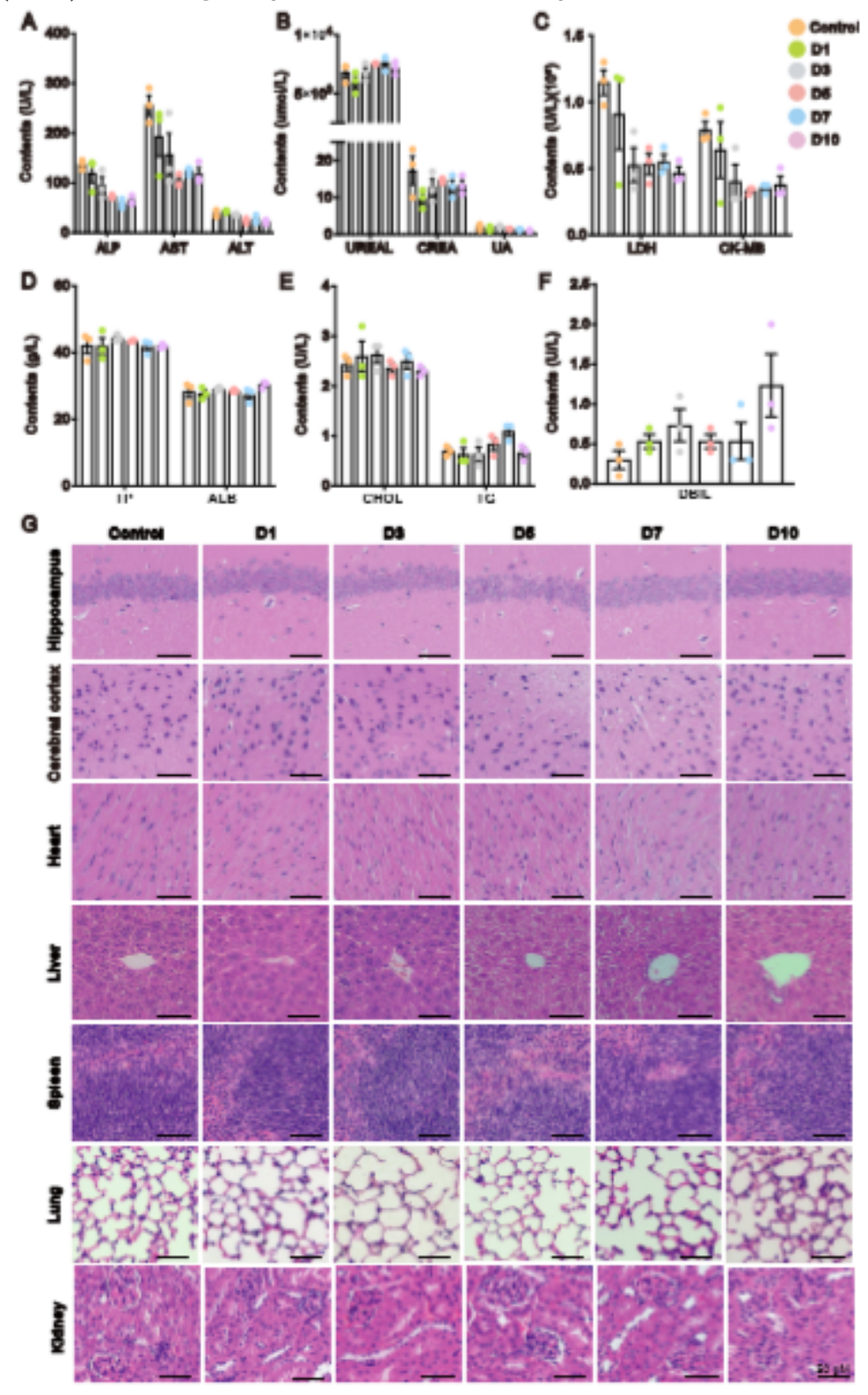

\section{Figure 2}

Cefepime shows no effect on serum chemistry and organ histology in mice. (a-f) Data are presented as the means \pm SEM. $n=3$ per group. Multiple comparison, ordinary one-way ANOVA. (g) Representative histological image of organs (HandE staining). 


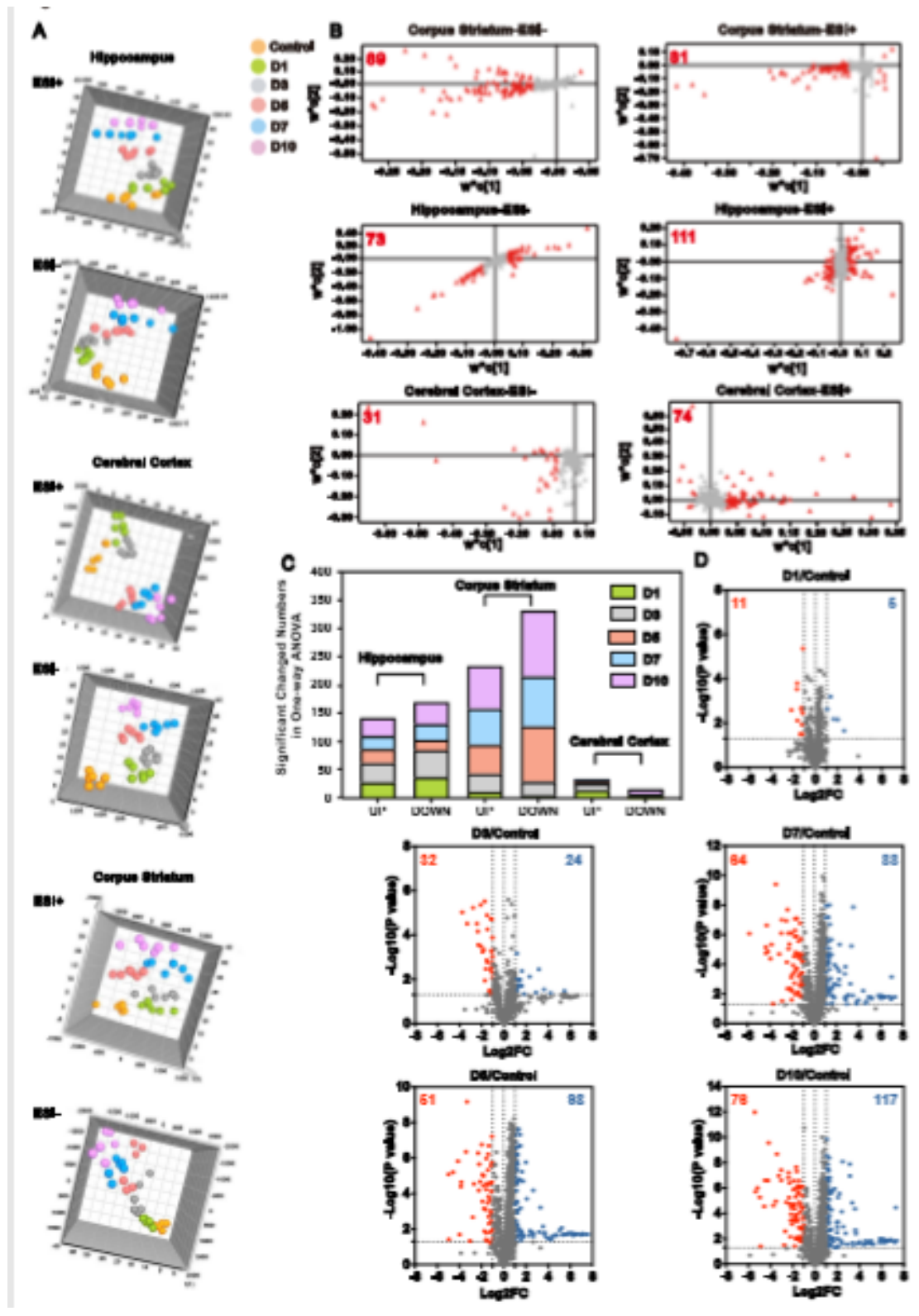

Figure 3

Pattern recognition analysis uncovers that cefepime alters the lipidome profile of corpus striatum. (a) 3D score scatter plots of OPLS-DA display the discrimination among the control and cefepime-treated groups under both positive and negative ion modes. (b) Loading plots based on the VIP value exhibit the putative ion peaks that mediate the discrimination of the control and cefepime-treated groups. Those VIP value above 1 or below 1 are labeled with red and light gray color, respectively. (c) Bar graph depicts the profoundly up-regulated (UP) and down-regulated (DOWN) ion peaks in the hippocampus, corpus striatum and cerebral cortex after cefepime exposure. (d) Volcano plots shows the profoundly affected ion peaks in corpus striatum after cefepime treatment. The volcano plots were constructed with $\mathrm{x}$-axis representing fold change level of ion peaks (logarithms of cefepime-treated group-to-control group) and yaxis representing the level of significance (logarithms of $p$ value). The significantly increased (-Log10 ( $p$ value) $>1.3$, Log2 (Fold-change) $>1$ ) and reduced (-Log10 ( $p$ value) $>1.3$, Log2 (Fold-change) <-1) ion peaks are marked with light blue and red color, respectively. 
A

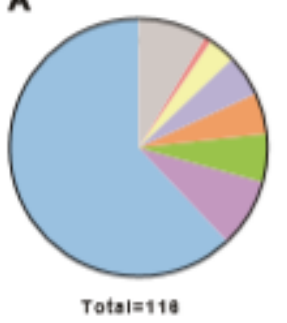

$\square .074$ OP

$\square .82 \%$ oL

$\square .084 \mathrm{PR}$

$\square .174$ FA

$\square .17 \%$ aP

$\square .45 \%$ बT

$\square 0.36 \%$ aL

$\square .42 \%$ other

c
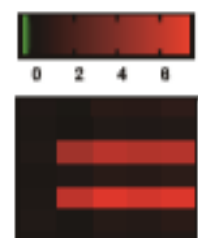

14:0r13:0

$14: 1 / 20: 0$
$18: 0,9-18: 0$

18:0.9:-18:0
$16: 0 / 22: 6$

20:0/16:0

14:0/20:0

20:4i18:0

18:0ris:2

18:0/22:

16:019:

20:419:0

22:6119:8

18:1/22:6

22:6/13:0

0-16:00-13:0 PC

18:06P. $18: 1$
20:8i1a:0

14:1/19:

13:0i1a: 8

22:A1P-18:1

22:613:1

22:2140:0

$22: 2 \pi 18: 0$
$13: 0 / 22: 2$

19:0r1a: 1

14:0/22:6

14:1/24:0

20:6/19:8

0.14:0118:0

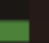

(

$18: 2 \pi 1: 0$
$14: 0 / 14: 0$
$20: 0 / 20: 3$$\quad P G$

20:0/20:8

$\frac{10: 0 / 22: 4}{20: 0 / 20: 2 n 6}$

14:0/2:2:2

14:0/20:

14:1/22:2

19:0/20:4ns DG

16:0n16:1

16:1/16:1
18:1nav18:1n

18:1กตั18:1n

22:4na/18:3n:
14:011::3
B 0000000000

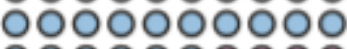



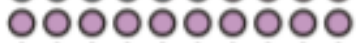

oอo-000000

วอำอำ.

००00000000

이이이잉

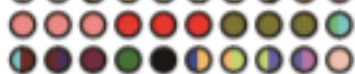

Totai=119

D
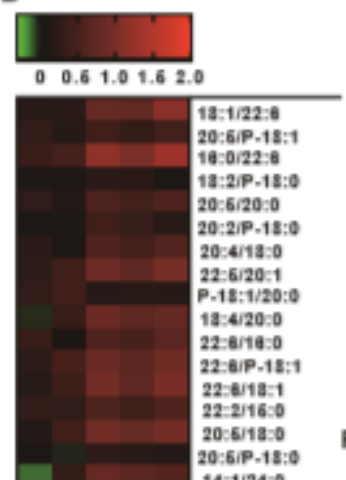

14:1/24:0

22:-122:

P.16:0/20:4

22:- $2020: 4$

22: $2122=0$

24:0/14:1

20:5/22:0

$22: 6 / 19: 0$
$0-20: 0 / 22: 4$

20:1/22:4

20:2/20:6

18:2/20:0

18:4/18:0

P-18:0122:4

$18: 0 / 2000$

$0-20: 0 / 17$ :

22:0/18:0

O-20:0/20:3 PS

$0.20: 4 / 18: 1$
$20: 2 / 21: 0$

$20: 9 / 21: 0$
$17: 2 / 22: 0$

$0-20: 0 / 18: 3$

18:14:e

D1 D: D6 D7 D10

PE

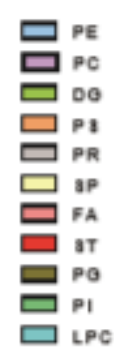

$\square$ aL

Masroesraal I

a-Phonyl-4-pontonal

Tormitomyoecphin A

Eonoxynol-s

$\square$ Torfensdins

Ethy1 1-(othyithio)othyi disuifide

$\square$ Distiohonio aold A

Sulfoglyoolithoenolato

$\square$ Austallde L

Monoothyinexyl phthalle aold

\section{E}
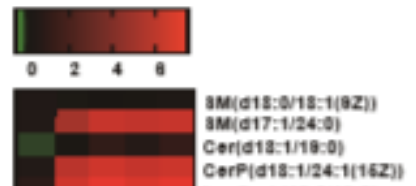

Corpidis:1/24:1162)

N-atoaroyleghingocine

CE(14:0)

MOOO(19:011a:2[0Z,122)!

12.Kotedooxyahello asid

Eploranome

Muzanzageain

Digarany:

Carbexy-40-mothyl-5a-ehelest:

19'-Hydroxy-alphs-foeotrianol

Ganoderto asid V

Molloellido $\mathrm{N}$

Toxin $\mathrm{T}_{2}$ totrol

so, $70,110-P$ hytanio acld

Polyexyothylene dielosto

Dieponurteanin A

asbadelin

9.10-OHOME

Oloamide

Macrosarpal।

3.Phonyl-4-pantena:

Tormitomy-pantona:

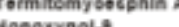

Tortengailine

Arsohidenile Aeld idas

Arsohidenie Adid (da)
Ethyl 1-(othylthlo/othyl dicuinde

Aulfoglyoeilitheeholats

Austallde $L$

Mosoethylinaxyl phthallo sold

PS 01 D8 06 07010

\section{Figure 4}

The aberrant glycerophospholipids contributes to the majority of cefepime-caused dysregulated lipids in corpus striatum. (a) Pie chart displays the proportion of identified lipid categories. All profoundly altered ion peaks (VIP $\geq 1, p<0.05)$ were identified and categorized through referring to the Lipid Maps

Database and the Human Metabolome Database. (b) $10 \times 10$ dot plot shows the ratio of each lipid species among all identified lipids. (c-e) Heatmap shows the changed level of differentially modified lipid species. Color bar represents the value of log2-fold changed level of each lipid. 


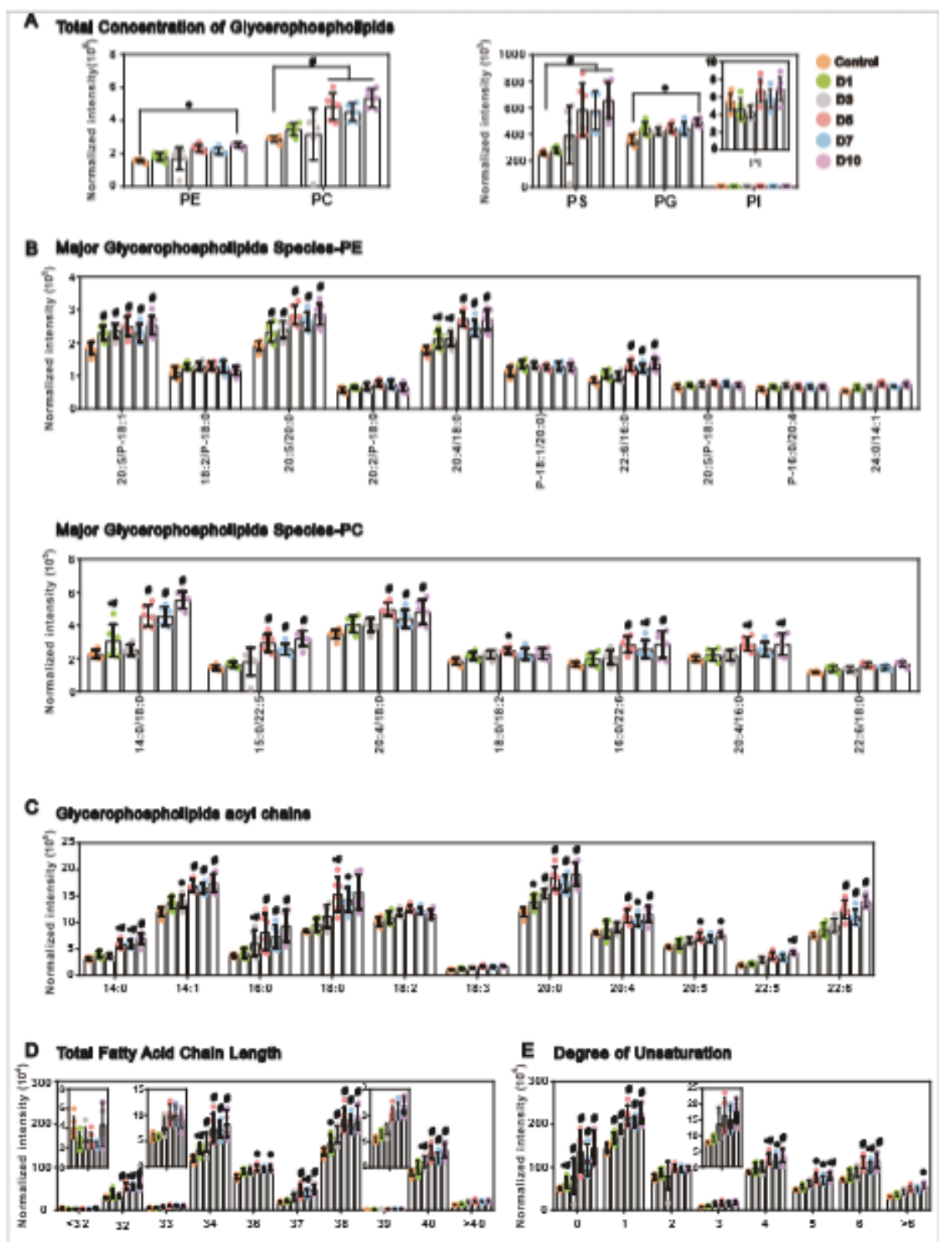

Figure 5

Cefepime exposure alter the abundance of glycerophospholipids as well as the length and unsaturation degree of acyl chain. (a) Bar graph displays the normalized intensity of PE, PC, PS, PG and PI after cefepime treatment for different time period. (b) Bar graph separately shows the normalized intensity of the major species of PC and PE after cefepime treatment for different time period. (c) Bar graph presents the normalized intensity of individual acyl chains associated with GP after cefepime treatment for different time period. ( $d$ and e) Bar graph displays the normalized intensity of length (d) and unsaturation degree (e) of acyl chain using all identified GP. Data are presented as means $\pm S D ; n=6$ for each group. Significance level: * $p<0.05, \& p<0.01, \# p<0.001$. 


\section{Figure 6}
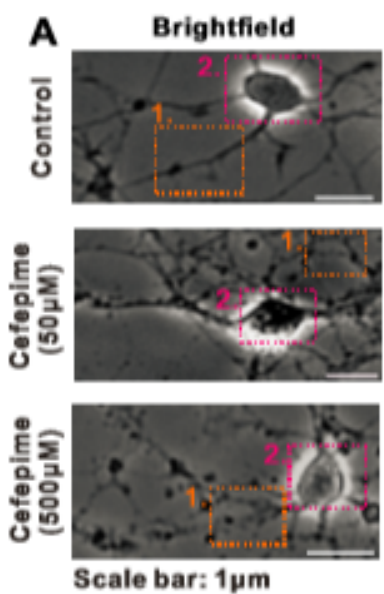

C Phalloidin/TOM20
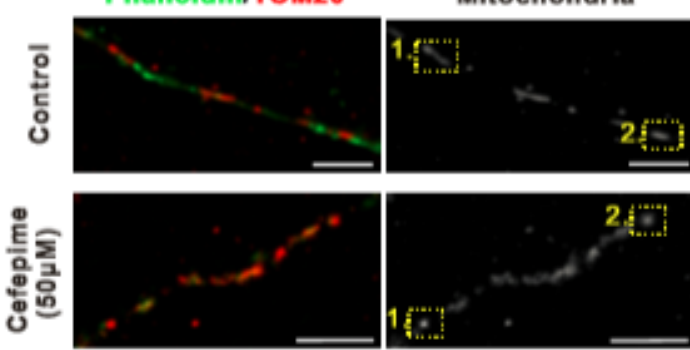

트ㅇㅡㅡㅇ

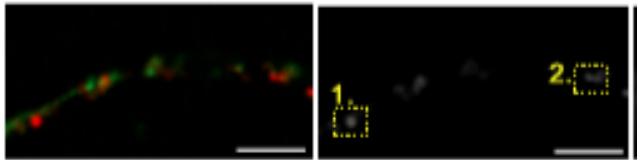

Scale bar: $5 \mu \mathrm{m}$

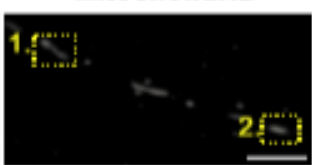

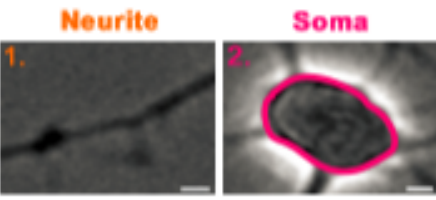
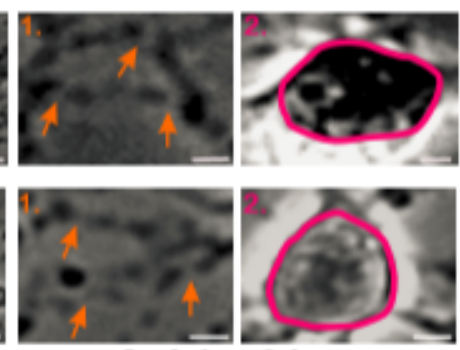

Scale bar: $0.1 \mu \mathrm{m}$
B

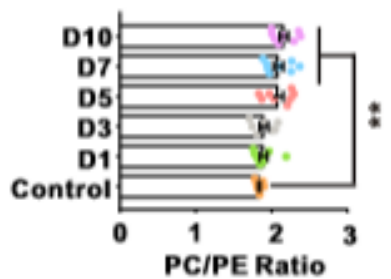

E

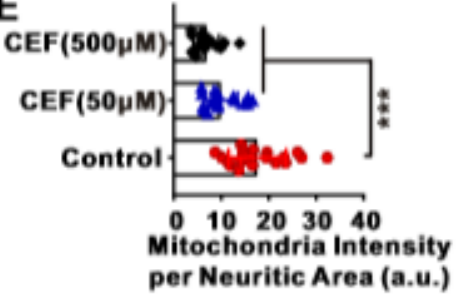

D

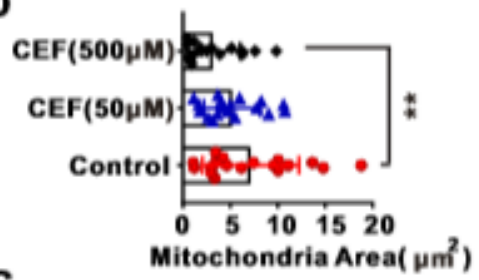

G

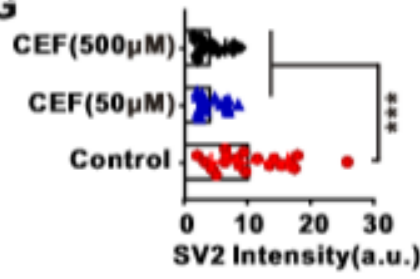

SV2

F

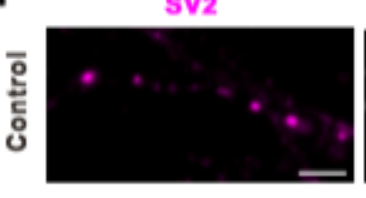

Synaptic Vesicle
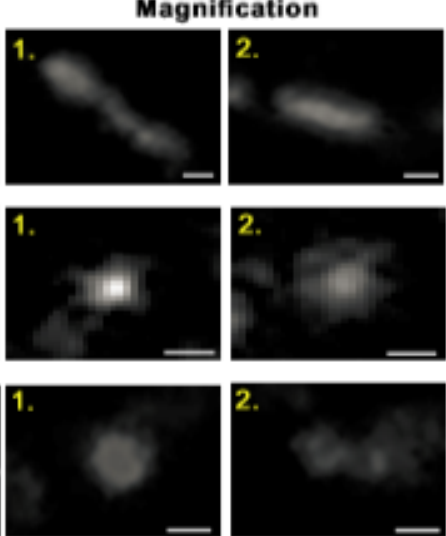

Scale bar: $0.1 \mu \mathrm{m}$
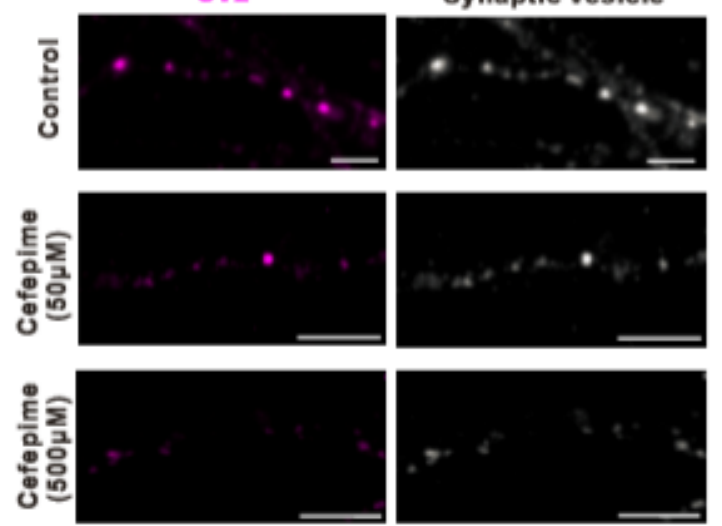

Scale bar: $5 \mu \mathrm{m}$

\section{Figure 6}

Cefepime treatment leads to the impairment of biological membrane-enriched structure in primary striatal neuron. (a) Brightfield images display the effect of cefepime on neuronal morphology. The magnified images in the right panel separately show the detailed morphology of neurite and soma area. (b) Bar graph presents the normalized intensity of PC/PE ratio in corpus striatum after cefepime treatment for indicated days. $n=6$ for each group. (c) The fluorescent images exhibit the effect of cefepime on mitochondrial morphology. Mitochondria were visualized via immunostaining the mitochondrial outer membrane protein TOM20. The profile of neurite was visualized through a fluorescent-labeled phalloidin, a probe specifically targeting to cytoskeleton actin. ( $d$ and e) Bar graph shows the quantified results of mitochondrial size (d) and mitochondrial density per neuritic area (e). Data are presented as means \pm SD; twenty individual neurons $(n=20)$ were used for quantification. Significance level: $* \star p<0.01$ and $* \star \star p$ $<0.001$. (f) The fluorescent images exhibit the effect of cefepime on synaptic vesicles through immunostaining with the synaptic vesicle protein SV2. (f) Bar graph shows the quantified density of synaptic vesicle per neuritic area. Data are presented as means \pm SD; twenty individual neurons $(n=20)$ were used for quantification. $* \star \star ~ p<0.001$.

\section{Supplementary Files}


This is a list of supplementary files associated with this preprint. Click to download.

- Onlinefloatimage1.png 